\title{
Effects of knockout of lincRNA-p21 on the proliferation, migration and invasion ability of HepG2 liver cancer cells
}

\author{
TONGSHAN WANG ${ }^{1 *}$, JUN LIU $^{2 *}$, SUIHUI LI ${ }^{3 *}$, ZHENGANG YUAN $^{4}$ and XIANGZHONG HUANG ${ }^{5}$ \\ ${ }^{1}$ Jiangsu Provincial People's Hospital Affiliated to Nanjing Medical University, Nanjing, Jiangsu 210029; \\ ${ }^{2}$ Department of Oncology, Affiliated Hospital of Nantong University, Nantong, Jiangsu 226001; \\ ${ }^{3}$ Department of Oncology, The First Hospital Affiliated to Guangzhou University of Chinese Medicine, \\ Guangzhou, Guangdong 510405; ${ }^{4}$ Department of Oncology, Eastern Hepatobiliary Hospital, Shanghai 200438; \\ ${ }^{5}$ Department of Interventional Radiology, Jiangsu Jiangyin People's Hospital, Wuxi, Jiangsu 214400, P.R. China
}

Received March 28, 2018; Accepted March 13, 2019

DOI: $10.3892 / 01.2019 .10201$

\begin{abstract}
Effects of long intergenic non-coding RNA (lincRNA)-p21 on the proliferation, migration and invasion ability of HepG2 liver cancer cells were assessed to explore the underlying mechanism. The lincRNA-p21 small interfering RNA (siRNA) lentivirus vector was constructed, transfected and screened to obtain a stable cell line, which constituted the experimental group. At the same time, the empty virus vector was transfected as the control group. The messenger RNA (mRNA) expression of lincRNA-p21 in cells was detected via reverse transcription-polymerase chain reaction (RT-PCR). The proliferation ability of cells was detected via Cell Counting kit-8 (CCK-8) assay. Transwell chamber experiment was used to observe cell migration and invasion ability. Compared with that in the control group, the mRNA expression level of lincRNA-p21 in cells in the experimental group was obviously decreased $(\mathrm{p}<0.05)$. Results of CCK- 8 showed that the proliferation ability of liver cancer cells was remarkably higher than that in the control group after knockout of lincRNA-p21 ( $\mathrm{p}<0.05)$. Results of the Transwell chamber experiment revealed that the invasion and migration ability of HepG2 cells in experimental group was markedly higher than that in control group $(\mathrm{p}<0.05)$. When lincRNA-p21 was inhibited, the proliferation, invasion and migration ability of HepG2 cells were significantly enhanced, and the apoptosis
\end{abstract}

Correspondence to: Dr Zhengang Yuan, Department of Oncology, Eastern Hepatobiliary Hospital, 225 Changhai Road, Shanghai 200438, P.R. China

E-mail: yuanzg_dr@126.com

Dr Xiangzhong Huang, Department of Interventional Radiology, Jiangsu Jiangyin People's Hospital, 35 Renmin East Road, Wuxi, Jiangsu 214400, P.R. China

E-mail: 1796309881@qq.com

*Contributed equally

Key words: long intergenic non-coding RNA-p21, HepG2, proliferation, apoptosis, invasion, migration rate was significantly decreased. Thus, lincRNA-p21 on the surface may play an inhibitory role in the occurrence, development and metastasis of liver cancer.

\section{Introduction}

Liver cancer is a common cancer worldwide, ranking sixth highest in incidence, with a fatality rate that is the third highest in highly lethal tumors globally. It is thus known as 'the king of cancers'. Among these tumors, hepatocellular carcinoma (HCC) accounts for $85-90 \%$ of deaths, and China is currently one of the countries with the most HCC deaths worldwide. HCC has a high degree of malignancy. Once the disease occurs, it progresses rapidly and the patient survival time is relatively short. The survival rate of HCC patients in the early stage in China is approximately $60 \%$. According to statistics the 5-year survival rate of HCC patients is approximately $33 \%$, which is mostly due to the occult onset of liver cancer and the lack of specific clinical manifestations in the early stage. In addition, patients were in the middle and advanced stage when diagnosed, and more importantly, there are few effective treatments (1-3).

At present, surgical resection remains the first choice of treatment. However, the vast majority of patients have already had near invasion or distant metastasis at the time of definite diagnosis; thus, surgical resection does not constitute a viable option. In addition, the recurrence rate of patients undergoing early surgical resection is high. Therefore, the identification of more effective methods for clinical diagnosis, treatment and prognosis monitoring is imperative $(4,5)$. Long intergenic non-coding RNAs (lincRNAs) refer to a class of long non-coding RNAs (lncRNAs) located between two coding genes. Currently, an increasing number of lincRNAs have been identified, such as lincRNA-p21, and an increasing number of studies have shown that lincRNA-p21 is involved in the development of a variety of human diseases, especially cancer $(6,7)$. At the same time, lincRNA-p21 is downregulated in many types of tumors compared with that in adjacent normal tissues, suggesting that lincRNA-p21 plays a key role as a tumor suppressor gene $(8,9)$. 
In this study, gene knockout technique was used to intervene in the expression of lincRNA-p21 in HepG2 cells, and the effects of lincRNA-p21 on the proliferation, migration and invasion ability of HepG2 cell line were studied.

\section{Materials and methods}

Experimental materials. The HepG2 liver cancer cell line was purchased from ATCC and preserved in our laboratory. The cells were identified as hepatocellular carcinoma cell lines by STR analysis, and there was no contamination. The eukaryotic expression plasmids containing lincRNA-p21 small interfering RNA (siRNA) sequence were constructed and prepared by GenScript Biotech Co., Ltd. (Nanjing, China). Dulbecco's modified Eagle's medium (DMEM) and fetal bovine serum were purchased from HyClone (HyClone: GE Healthcare, Logan, UT, USA). TRIzol reagent kit, messenger RNA (mRNA) extraction kit, complementary DNA (cDNA) synthesis kit and reverse transcription-polymerase chain reaction (RT-PCR) amplification kit were purchased from Invitrogen (Invitrogen: Thermo Fisher Scientific, Inc., Carlsbad, CA, USA). Cell Counting kit-8 (CCK-8) and apoptosis detection kit were purchased from Beyotime Institute of Biotechnology (Haimen, China). Transwell chamber and Matrigel were purchased from Corning, Inc. (Corning, NY, USA). Fluorescent quantitative PCR instrument was purchased from Applied Biosystems (Applied Biosystems: Thermo Fisher Scientific, Inc., Foster City, CA, USA). Flow cytometer was purchased from BD Biosciences (Franklin Lakes, NJ, USA). Fluorescence microscope was purchased from Olympus Corp. (Tokyo, Japan).

The study was approved by the Ethics Committee of Jiangsu Provincial People's Hospital Affiliated to Nanjing Medical University (Nanjing, China).

Construction and transfection of lincRNA-p21 siRNA lentivirus vector. The constructed recombinant plasmids containing lincRNA-p21 siRNA were transfected into 293T cells. After culture, cell supernatants rich in virus particles were collected, filtered and stored at $-80^{\circ} \mathrm{C}$ for standby application. After $40-60 \%$ of the cells were fused, HepG 2 cells in the logarithmic growth phase were added with the recombinant lentivirus vector containing lincRNA-p21 siRNA (experimental group) and empty virus vector (control group), respectively. The cells were incubated in an incubator with $5 \% \mathrm{CO}_{2}$ at $37^{\circ} \mathrm{C}$ for $24 \mathrm{~h}$, and then the medium was replaced. Stable cells were obtained for expansion culture by G418 screening.

Detection of lincRNA-p21 mRNA expression via RT-PCR. The transfected HepG2 cells were lysed with TRIzol reagent and the total cell RNA was extracted according to the instructions of mRNA extraction kit to measure RNA concentration. The total RNA was reversely transcribed into cDNA according to the cDNA synthesis kit. lincRNA-p21 primers were designed, including lincRNA-p21 forward, 5'-CCCGGGCTTGTC TTTTGTT-3' and reverse, 5'-GAGTGGGTGGCTCACTCT TCTG-3'; glyceraldehyde-3-phosphate dehydrogenase (GAPDH) forward, 5'-CTGGGCTACACTGAGCACC-3' and reverse, 5'-AAGTGGTCGTTGAGGGCAATG-3'. According to the instructions of RT-PCR amplification kit, SYBR-Green assay was used for quantitative PCR detection. Reaction conditions: pre-denaturation at $95^{\circ} \mathrm{C}$ for $15 \mathrm{~min}$, denaturation at $95^{\circ} \mathrm{C}$ for $15 \mathrm{sec}$, annealing at $60^{\circ} \mathrm{C}$ for $30 \mathrm{sec}$, fluorescence collection, extension at $72^{\circ} \mathrm{C}$ for $30 \mathrm{sec}$, a total of 40 cycles, data reading and quantitative analysis.

Detection of cell proliferation ability via CCK-8 assay. Cells in experimental and control group were digested with trypsin. The concentration of cells was adjusted to $5 \times 10^{4} / \mathrm{ml}$ with DMEM containing 10\% fetal bovine serum. A total of $100 \mu 1$ of cell suspension was inoculated into a 96-well plate and cultured at $37^{\circ} \mathrm{C}$ for $12,24,48$, and $72 \mathrm{~h}$, respectively. A total of $10 \mu \mathrm{l}$ CCK- 8 was added and gently mixed well using an oscillator, followed by cultivation in an incubator at $37^{\circ} \mathrm{C}$ for $4 \mathrm{~h}$. Double-wavelength measurements were performed on a microplate reader with a detection wavelength of $490 \mathrm{~nm}$ and a reference wavelength of $650 \mathrm{~nm}$. The cell proliferation curve was plotted based on optical density (OD) values.

Detection of apoptosis by flow cytometry. Logarithmic growth phase cells in experimental and control group were digested with trypsin without ethylenediaminetetraacetic acid (EDTA), collected, and washed with phosphate-buffered saline (PBS) twice. A total of $5 \times 10^{5}$ cells were collected in each tube, and $500 \mu \mathrm{l}$ binding buffer was added to $100 \times 10^{1}$ dyeing buffer for cell resuspension. Then, $5 \mu 1$ Annexin V and $10 \mu 1$ propidium iodide (PI) dyeing liquor were added, respectively, and mixed well, followed by reaction at room temperature in the dark for $15 \mathrm{~min}$. Cell apoptosis was detected by flow cytometry. Three parallel samples were set up in each experimental group. Apoptosis rate $(\%)=($ Annexin $\mathrm{V}+\mathrm{PI}+$ cell number + Annexin V + PI - cell number)/10,000 x 100\%

Transwell chamber migration experiment. The Transwell chamber was placed in a 24 -well plate. HepG2 cells in the experimental and control groups were digested with trypsin and pipetted into single cell suspension, which was then washed with PBS twice. Cells were resuspended in serum-free medium and the cell concentration was adjusted to $5 \times 10^{5} / \mathrm{ml}$. A total of $100 \mathrm{ml}$ suspension was taken and inoculated in the Transwell chamber. In the lower chamber, $500 \mu \mathrm{l}$ DMEM containing $10 \%$ fetal bovine serum was added for cultivation in an incubator at $37^{\circ} \mathrm{C}$ for $20-24 \mathrm{~h}$. The Transwell chamber was taken out and washed with PBS twice, followed by fixation with 5\% glutaraldehyde for $30 \mathrm{~min}$, washing with PBS twice, staining with $0.1 \%$ crystal violet for $20 \mathrm{~min}$ and washing with PBS twice. The unmigrated cells on the upper surface were erased with cotton ball and the number of perforating cells was calculated by randomly taking images of 8-10 visual fields by an optical microscope at a magnification of $\times 100$.

Transwell chamber invasion experiment. The Transwell chamber was placed in a 24-well plate. BD Matrigel was diluted with serum-free DMEM at 1:6 and $100 \mu \mathrm{l}$ of diluted BD Matrigel was added into the Transwell chamber, followed by cultivation in an incubator at $37^{\circ} \mathrm{C}$ for $6 \mathrm{~h}$. BD Matrigel was washed with serum-free medium once. HepG2 cells in experimental and control group were digested with trypsin and pipetted into single cell suspension, which was then washed with PBS twice. Cells were resuspended in serum-free 


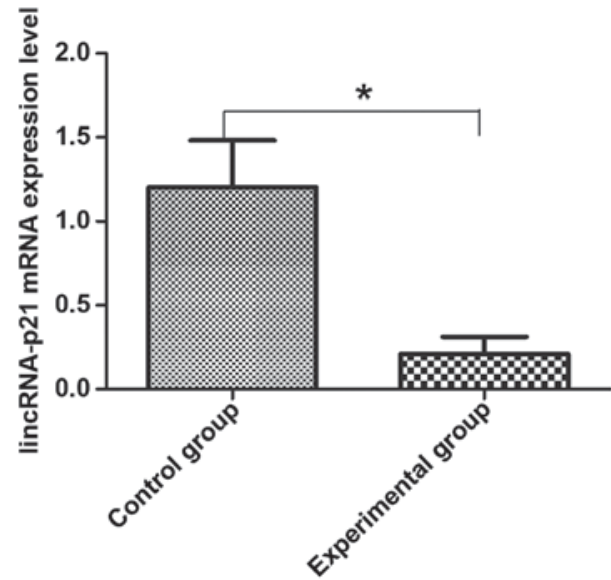

Figure 1. Effects of siRNA interference with lincRNA-p21. siRNA, small interfering RNA; lincRNA, long intergenic non-coding RNA. Control group vs. experimental group, ${ }^{\mathrm{P}} \mathrm{P}<0.05$.

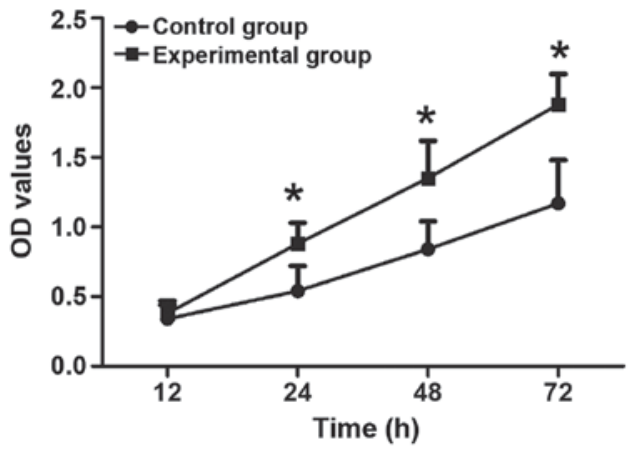

Figure 2. Effects of lincRNA-p21 knockout on the proliferation of HepG2 cells. lincRNA, long intergenic non-coding RNA. Control group vs. experimental group at different time points, ${ }^{*} \mathrm{P}<0.05$.

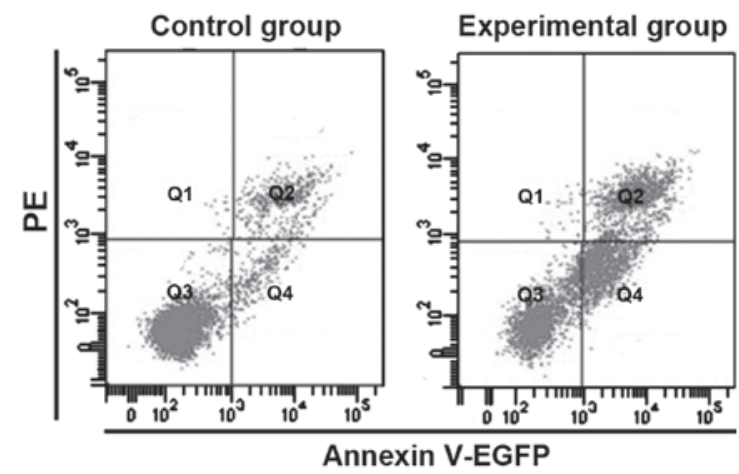

Figure 3. Effects of lincRNA-p21 knockout on the apoptosis of HepG2 cells. lincRNA, long intergenic non-coding RNA.

medium and the cell concentration was adjusted to $5 \times 10^{5} / \mathrm{ml}$. A total of $100 \mathrm{ml}$ suspension was taken and inoculated into the Transwell chamber. In the lower chamber, $500 \mu$ l DMEM containing $10 \%$ fetal bovine serum was added for cultivation in an incubator at $37^{\circ} \mathrm{C}$ for $20-24 \mathrm{~h}$. The Transwell chamber was taken out and washed with PBS twice, followed by fixation with $5 \%$ glutaraldehyde for $30 \mathrm{~min}$, washing with PBS twice, staining with $0.1 \%$ crystal violet for $20 \mathrm{~min}$, and washing with PBS twice. The upper layer cells of BD Matrigel
Table I. Effects of lincRNA-p21 knockout on the proliferation of HepG2 cells.

\begin{tabular}{lcccc}
\hline Group & $12 \mathrm{~h}$ & $24 \mathrm{~h}$ & $48 \mathrm{~h}$ & $72 \mathrm{~h}$ \\
\hline Control & $0.34 \pm 0.10$ & $0.54 \pm 0.18$ & $0.84 \pm 0.20$ & $1.17 \pm 0.31$ \\
Experimental & $0.38 \pm 0.09$ & $0.88 \pm 0.15$ & $1.35 \pm 0.27$ & $1.88 \pm 0.22$ \\
P-value & 0.109 & 0.043 & 0.031 & 0.0102 \\
\hline
\end{tabular}

lincRNA, long intergenic non-coding RNA.

Table II. Comparisons of cell migration and invasion number between two groups (cells/HP).

\begin{tabular}{lcc}
\hline Group & Cell migration no. & Cell invasion no \\
\hline Control & $90.7 \pm 14.8$ & $30.6 \pm 8.6$ \\
Experimental & $215.3 \pm 18.9^{\mathrm{a}}$ & $87.5 \pm 10.3^{\mathrm{a}}$
\end{tabular}

${ }^{\mathrm{a}} \mathrm{P}<0.05$ vs. control group.

and microporous membrane were erased with cotton ball. The number of perforated cells was calculated by randomly taking images of 8-10 visual fields by an optical microscope at a magnification of $\mathrm{x} 100$.

Statistical analysis. Statistical Product and Service Solutions (SPSS) 17.0 statistical software was used for statistical analysis. Enumeration data are expressed as means \pm SD. Analysis of variance was used for multigroup comparison as well as least significant difference (LSD) post hoc test. The t-test was used for comparison between two groups. $\mathrm{P}<0.05$ indicates that the difference was statistically significant.

\section{Results}

Detection of knockout effects of lincRNA-p21 mRNA via $R T-P C R$. Compared with empty transfected HepG2 cells in control group, the mRNA expression of lincRNA-p21 in cells in the experimental group was significantly decreased, indicating that the transfection of lincRNA-p21 siRNA can effectively interfere with the expression level of lincRNA-p21 in HepG2 cells, which laid the foundation for the next experiment (Fig. 1).

Effects of lincRNA-p21 knockout on the proliferation of HepG 2 cells. CCK-8 assay was used to detect the relationship between the downregulation of lincRNA-p21 expression and HepG2 cell proliferation. The results are shown in Fig. 2 and Table I. Compared with that in control group, the cell proliferation ability in the experimental group was obviously enhanced, and the difference was obvious from $24 \mathrm{~h}(\mathrm{p}<0.05)$, indicating that the downregulation of lincRNA-p21 expression can promote the proliferation of HepG2 cells.

Effects of lincRNA-p21 knockout on apoptosis of HepG2 cells. The apoptosis rate of HepG2 cells in logarithmic growth 
phase in experimental and control group was detected via flow cytometry. As shown in Fig. 3, the apoptotic rates in the experimental and control group were 5.21 \pm 1.43 and $26.33 \pm 5.13$, respectively. Compared with that in control group, the apoptosis rate in experimental group was significantly decreased $(\mathrm{p}<0.05)$, indicating that knockout lincRNA-p21 can inhibit HepG2 cell apoptosis.

Effects of lincRNA-p21 knockout on the migration and invasion ability of HepG2 cells. The relationship between the downregulation of lincRNA-p21 expression and the migration and invasion of HepG 2 cells was detected via Transwell assay. The results revealed that after crystal violet staining, cells in control and experimental group passing through chamber filter membrane were observed in both migration and invasion experiments. The number of cells that passed through the membrane in experimental group was significantly higher than that in control group $(\mathrm{p}<0.05)$, indicating that knockout of lincRNA-p21 can effectively improve the migration and invasion ability of HepG2 cells (Table II).

\section{Discussion}

Liver cancer is the most common malignant tumor in China, whose morbidity and mortality accounts for $\sim 50 \%$ of those in the whole world, making China one of the regions with the highest incidence of liver cancer in the world. Since liver cancer patients often do not have specific symptoms or signs, it is difficult to diagnose early. Therefore, in clinic, many patients with liver cancer have entered the middle or late stages when they are diagnosed. For patients with middle or late liver cancer, there is still no effective clinical treatment $(10,11)$. It is noteworthy that liver cancer is characterized by high invasiveness, early metastasis, high recurrence rate, poor prognosis, insensitivity to radiotherapy, low effective rate of chemotherapy, and extreme resistance to drugs. Its mortality rate has remained high, and metastasis is the main reason for the high mortality rate. Therefore, it is of great significance to study the mechanism of tumor metastasis and explore effective treatment methods to control the metastasis and recurrence of liver cancer and improve the survival rate and quality of life of liver cancer patients (12-14). IncRNAs and their subtype lincRNAs are biologically functional non-coding RNAs discovered in recent years. IncRNA is a type of RNA molecule that does not encode proteins and has a transcript of $>200 \mathrm{nt}$ in length. It regulates gene expression at multiple levels in the form of RNA $(15,16)$. Research has manifested that lncRNAs play an important role in the formation and development of tumors. Therefore, in-depth study of the function and mechanism of lncRNA in tumors will help find potential target genes for the treatment of tumors.

p53 is a key molecule that regulates a variety of biological behaviors of cells, and plays an important role in the repair of damaged DNA, apoptosis, proliferation and other regulatory aspects. lincRNA-p21 is found to be a kind of lncRNA most closely related to p53 expression. p53 binds directly to the upstream regulatory region of lincRNA-p21 and regulates its transcriptional level $(17,18)$. Clinical studies have proven that the low expression of lincRNA-p21 predicts a lower survival rate of cancer patients, and it can be used in the future as a new molecular marker for the diagnosis and prognosis to stimulate new research directions and treatment options.

In order to further clarify the role of lincRNA-p21 in the progression of liver cancer, RNA interference (RNAi) technology was used to interfere with the expression of lincRNA-p21 in HCC HepG2 cells in this study. The results revealed that after the expression of lincRNA-p21 was successfully inhibited, the proliferation ability of HCC cell line HepG2 was obviously enhanced, flow cytometry showed a significant decrease in the apoptosis ability, and the results of Transwell chamber experiment demonstrated that the invasion and migration ability were remarkably increased. These results further confirmed that lincRNA-p21 promotes apoptosis and inhibits the proliferation, invasion and metastasis ability of HCC cells, pointing out a direction of further study on lincRNA-p21 transcriptional regulation mechanism and new drugs for liver cancer.

\section{Acknowledgements}

Not applicable.

\section{Funding}

No funding was received.

\section{Availability of data and materials}

The datasets used and/or analyzed during the present study are available from the corresponding author on reasonable request.

\section{Authors' contributions}

TW, JL, SL, ZY and XH were responsible for the conception and study design. TW, JL, SL, ZY and XH were responsible for the collection and assembly of data. TW, JL and SL were responsible for data analysis and interpretation. TW, JL, SL, ZY and $\mathrm{XH}$ were responsible for the writing and final approval of manuscript. All authors read and approved the final manuscript.

\section{Ethics approval and consent to participate}

The study was approved by the Ethics Committee of Jiangsu Provincial People's Hospital Affiliated to Nanjing Medical University (Nanjing, China).

\section{Patient consent for publication}

Not applicable.

\section{Competing interests}

The authors declare that they have no competing interests.

\section{References}

1. Sun T, Liu H and Ming L: Multiple roles of autophagy in the sorafenib resistance of hepatocellular carcinoma. Cell Physiol Biochem 44: 716-727, 2017. 
2. Pinter M, Weinmann A, Wörns MA, Hucke F, Bota S, Marquardt JU, Duda DG, Jain RK, Galle PR, Trauner M, et al: Use of inhibitors of the renin-angiotensin system is associated with longer survival in patients with hepatocellular carcinoma. United European Gastroenterol J 5: 987-996, 2017.

3. Sanduzzi Zamparelli M, Rocco A, Compare D and Nardone G: The gut microbiota: A new potential driving force in liver cirrhosis and hepatocellular carcinoma. United European Gastroenterol J 5: 944-953, 2017.

4. An C, Hu ZL, Liang P, Cheng ZG, Han ZY, Yu J and Liu FY: Ultrasound-guided percutaneous microwave ablation vs. surgical resection for thoracoabdominal wall implants from hepatocellular carcinoma: Intermediate-term results. Int J Hyperthermia 21: $1-10,2017$.

5. Chen X, Jiang W, Yue C, Zhang W, Tong C, Dai D, Cheng B Huang $\mathrm{C}$ and Lu L: Heparanase contributes to trans-endothelial migration of hepatocellular carcinoma cells. J Cancer 8: 3309-3317, 2017.

6. Chen S, Liang H, Yang H, Zhou K, Xu L, Liu J, Lai B, Song L, Luo H, Peng J, et al: LincRNa-p21: Function and mechanism in cancer. Med Oncol 34: 98, 2017.

7. Chillón I and Pyle AM: Inverted repeat Alu elements in the human lincRNA-p21 adopt a conserved secondary structure that regulates RNA function. Nucleic Acids Res 44: 9462-9471, 2016

8. Shen Y, Liu Y, Sun T and Yang W: LincRNA-p21 knockdown enhances radiosensitivity of hypoxic tumor cells by reducing autophagy through HIF-1/Akt/mTOR/P70S6K pathway. Exp Cell Res 358: 188-198, 2017.

9. Chen Y, Wei G, Xia H, Yu H, Tang Q and Bi F: Down regulation of lincRNA-p21 contributes to gastric cancer development through Hippo-independent activation of YAP. Oncotarget 8: 63813-63824, 2017.

10. Wang JY, Fang M, Boye A, Wu C, Wu JJ, Ma Y, Hou S, Kan Y and Yang Y: Interaction of microRNA-21/145 and Smad3 domain-specific phosphorylation in hepatocellular carcinoma. Oncotarget 8: 84958-84973, 2017.

11. Feng LH, Wang H, Dong H, Zhu YY and Cong WM: The stromal morphological changes for differential diagnosis of uninodular high-grade dysplastic nodule and well-differentiated small hepatocellular carcinoma. Oncotarget 8: 87329-87339, 2017.
12. Ye JZ, Chen JZ, Li ZH, Bai T, Chen J, Zhu SL, Li LQ and Wu FX: Efficacy of postoperative adjuvant transcatheter arterial chemoembolization in hepatocellular carcinoma patients with microvascular invasion. World J Gastroenterol 23: 7415-7424, 2017.

13. Sim HW and Knox J: Hepatocellular carcinoma in the era of immunotherapy. Curr Probl Cancer 42: 40-48, 2018.

14. Honda H, Takamura M, Yamagiwa S, Genda T, Horigome R, Kimura N, Setsu T, Tominaga K, Kamimura H, Matsuda Y, et al: Overexpression of a disintegrin and metalloproteinase 21 is associated with motility, metastasis, and poor prognosis in hepatocellular carcinoma. Sci Rep 7: 15485, 2017.

15. Ding G, Peng Z, Shang J, Kang Y, Ning H and Mao C: LincRNA-p21 inhibits invasion and metastasis of hepatocellular carcinoma through miR-9/E-cadherin cascade signaling pathway molecular mechanism. OncoTargets Ther 10: 3241-3247, 2017.

16. Jia M, Jiang L, Wang YD, Huang JZ, Yu M and Xue HZ: lincRNA-p21 inhibits invasion and metastasis of hepatocellular carcinoma through Notch signaling-induced epithelial-mesenchymal transition. Hepatol Res 46: 1137-1144, 2016.

17. Yu F, Guo Y, Chen B, Shi L, Dong P, Zhou M and Zheng J: LincRNA-p21 Inhibits the Wnt/ $\beta$-catenin pathway in activated hepatic stellate cells via sponging microRNA-17-5p. Cell Physiol Biochem 41: 1970-1980, 2017

18. Castellano JJ, Navarro A, Viñolas N, Marrades RM, Moises J, Cordeiro A, Saco A, Muñoz C, Fuster D, Molins L, et al: LincRNA-p21 impacts prognosis in resected non-small cell lung cancer patients through angiogenesis regulation. J Thorac Oncol 11: 2173-2182, 2016.

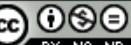

This work is licensed under a Creative Commons Attribution-NonCommercial-NoDerivatives 4.0 International (CC BY-NC-ND 4.0) License. 